\title{
Rare metastatic pancreatic tumors from lung cancer with cystic changes resembling intraductal papillary mucinous neoplasm: a case report
}

\author{
Akira Watanabe ${ }^{1}$ D , Norifumi Harimoto ${ }^{1,2^{*}}$, Takahiro Yamanaka ${ }^{1}$, Norihiro Ishii ${ }^{1}$, Mariko Tsukagoshi ${ }^{1,3}$, \\ Takamichi Igarashi ${ }^{1}$, Norio Kubo ${ }^{1}$, Kenichiro Araki ${ }^{1}$, Aya Suzuki ${ }^{5}$, Kenichiro Hara ${ }^{4}$ and Ken Shirabe ${ }^{1}$
}

\begin{abstract}
Background: Metastatic pancreatic tumors from lung cancer (MPTLC) constitute 3\% of all metastatic pancreatic tumors. We present an extremely rare case of cystic MPTLC that was difficult to distinguish from intraductal papillary mucinous neoplasm (IPMN).

Case presentation: The patient was a 74-year-old woman who underwent lobectomy of lung cancer 2 years before presentation to our hospital. She was referred to our department for resection of cystic pancreatic tumors, which were diagnosed as IPMN with high-risk stigmata. Abdominal computed tomography (CT) showed a 37-mmwide cystic tumor with a contrasted solid nodule in the pancreatic head and a 17-mm-wide cystic tumor in the pancreatic tail. We performed a total pancreatectomy for these lesions. According to histopathological and immunohistochemical findings, the tumors were diagnosed as metastatic pancreatic tumors from lung cancer.

Conclusion: In this case, the cystic morphology was formed by eosinophilic secretions from tumor cells, and it was difficult to distinguish from IPMN with high-risk stigmata. We consider this case, based on the variable clinical findings, an extremely rare variant of MPTLC.
\end{abstract}

Keywords: Metastatic pancreatic tumor from lung cancer (MPTLC), Cystic tumor, Intraductal papillary mucinous neoplasm (IPMN), High-risk stigmata

\section{Background}

Metastatic pancreatic tumors from lung cancer (MPTLC) constitute $3 \%$ of all metastatic pancreatic tumors [1]. Although MPTLC is mainly treated with chemotherapy, pancreatectomy is sometimes performed in cases of solitary or metachronal metastasis. MPTLC is reported to present as hypovascular or ring-enhancing lesions on

\footnotetext{
* Correspondence: nharimotoh1@gunma-u.ac.jp

'Department of General Surgical Science, Division of Hepatobiliary and

Pancreatic Surgery, Graduate School of Medicine, Gunma University, 3-39-15 Showamachi, Maebashi, Gunma 371-8511, Japan

${ }^{2}$ Department of General Surgical Science, Division of Hepatobiliary and Pancreatic Surgery, Graduate School of Medicine, Gunma University, 3-39-22

Showamachi, Maebashi, Gunma 371-8511, Japan

Full list of author information is available at the end of the article
}

imaging findings [2], but it is difficult to distinguish from primary pancreatic cancer. Because MPTLC typically forms solid tumors, cystic changes of MPTLC are extremely rare. Herein, we reported a case of cystic MPTLC, which was difficult to distinguish from intraductal papillary mucinous neoplasm (IPMN).

\section{Case presentation}

The patient was a 74-year-old female who underwent left lower lobectomy for lung cancer 2 years before presenting to our institution. The histological type was adenocarcinoma, with a pathological staging of T4N1M0 stage IIIA (Union for International Cancer Control: UICC 8th ed) [3]. One year after lobectomy, cystic

\section{Springer Open}

(๑) The Author(s). 2020 Open Access This article is licensed under a Creative Commons Attribution 4.0 International License, which permits use, sharing, adaptation, distribution and reproduction in any medium or format, as long as you give appropriate credit to the original author(s) and the source, provide a link to the Creative Commons licence, and indicate if changes were made. The images or other third party material in this article are included in the article's Creative Commons licence, unless indicated otherwise in a credit line to the material. If material is not included in the article's Creative Commons licence and your intended use is not permitted by statutory regulation or exceeds the permitted use, you will need to obtain permission directly from the copyright holder. To view a copy of this licence, visit http://creativecommons.org/licenses/by/4.0/. 


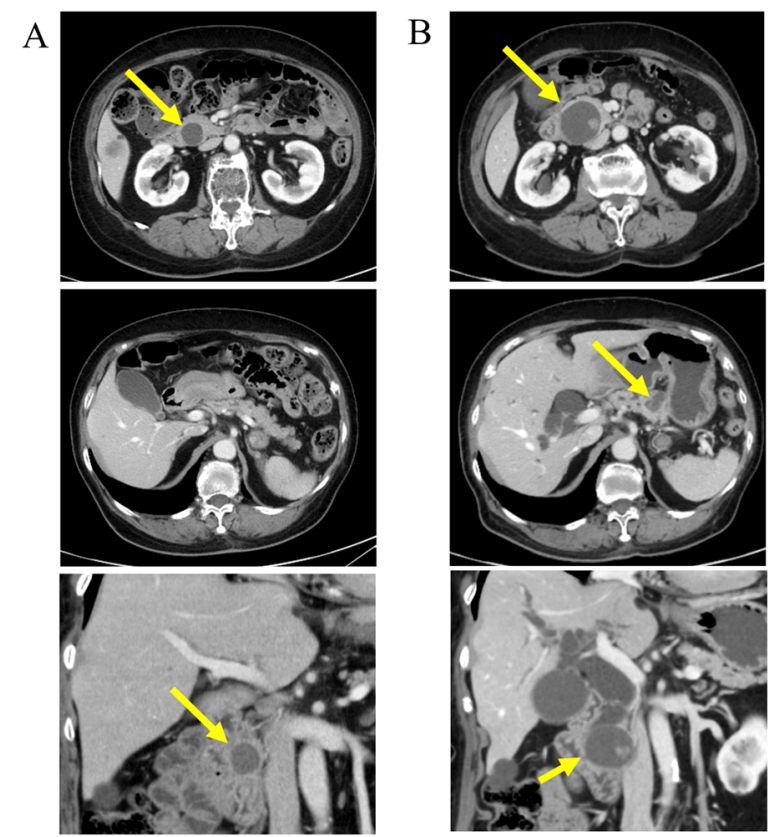

Fig. 1 Enhanced computed tomography findings. a CT findings at 1 year after lobectomy for primary lung cancer. A 20-mm cystic tumor was detected on the pancreatic head, with no tumor on the pancreatic body. $\mathbf{b} C T$ findings at 2 year after lobectomy. The cystic tumor on the pancreatic head gradually increased from $20 \mathrm{~mm}$ to $37 \mathrm{~mm}$ and showed a contrasted solid nodule inside the cystic tumor. The cystic tumor was also indicated at the pancreatic body

lesions appeared on the head and tail of the pancreas, diagnosed as IPMN. The cystic tumor on the pancreatic head gradually increased from 20 to $37 \mathrm{~mm}$ in 1 year and showed a contrasted solid nodule inside the cystic tumor (Fig. 1). The patient was referred to our department for surgery because the tumor was considered IPMN with high-risk stigmata. Her blood test results were as follows: carcinoembryonic antigen, $2.4 \mathrm{ng} / \mathrm{mL}$ (normal range, < $5.0 \mathrm{ng} / \mathrm{mL}$ ); carbohydrate antigen 19-9, $38 \mathrm{U} / \mathrm{mL}$ (normal range, < $15 \mathrm{U} / \mathrm{mL}$ ); DUPAN-2, $39 \mathrm{U} /$ $\mathrm{mL}$ (normal range, < $150 \mathrm{U} / \mathrm{mL}$ ); and SPAN-1, $29.8 \mathrm{U} /$
$\mathrm{mL}$ (normal range, $<30 \mathrm{U} / \mathrm{mL}$ ). Abdominal computed tomography (CT) showed a $37-\mathrm{mm}$ cystic tumor with a contrasted solid nodule at the pancreatic head and a 17$\mathrm{mm}$ cystic tumor at the pancreatic tail. Endoscopic ultrasonography (EUS) revealed that the cystic tumor at the head was a $35-\mathrm{mm}$ solitary cyst with a $24-\mathrm{mm}$ mural nodule, and the cystic tumor at the tail was a $20-\mathrm{mm}$ solitary cyst with a $10-\mathrm{mm}$ mural nodule. The main pancreatic duct had no extension. Although we had confirmed that the cystic tumor and main pancreatic duct were close, we could not define the link between the main pancreatic duct and the cyst (Fig. 2). ${ }^{18} \mathrm{~F}$-fluorodeoxyglucose positron-emission tomography (FDG-PET) showed FDG uptake (SUV max 1.9) at the lesion in the pancreatic head. No evidence of metastasis from other organs was observed (Fig. 3). Magnetic resonance imaging (MRI) could not be performed because of a cardiac pacemaker. The patient developed jaundice because the pancreatic head tumor excluded the common bile duct. From these results, we diagnosed the tumors as IPMN with high-risk stigmata because of jaundice and a contrasted mural nodule. We performed a total pancreatectomy for the two lesions after bile duct drainage by endoscopic retrograde cholangiopancreatography (ERCP). Because we performed ERCP on emergency, we could not perform brushing cytology or pancreatic juice cytology for food residue in the stomach and duodenum. The tumors were solitary cysts with papillary lesions at the pancreatic head and tail. Histopathological findings showed that tumor cells had papillary components without mucus production (Fig. 4a, b, c). Moreover, a small tumor lesion was also microscopically detected at the pancreatic tail. Immunohistochemical analysis showed positive results for TTF-1, Napsin A, and CK7, but CK20 did not present significant staining (Fig. 4d, e, f), and these findings indicated this tumor to be lung cancer metastasis rather than IPMN. The histological findings were similar to those of the existing lung adenocarcinoma resected 2 years before now (Fig. 5).
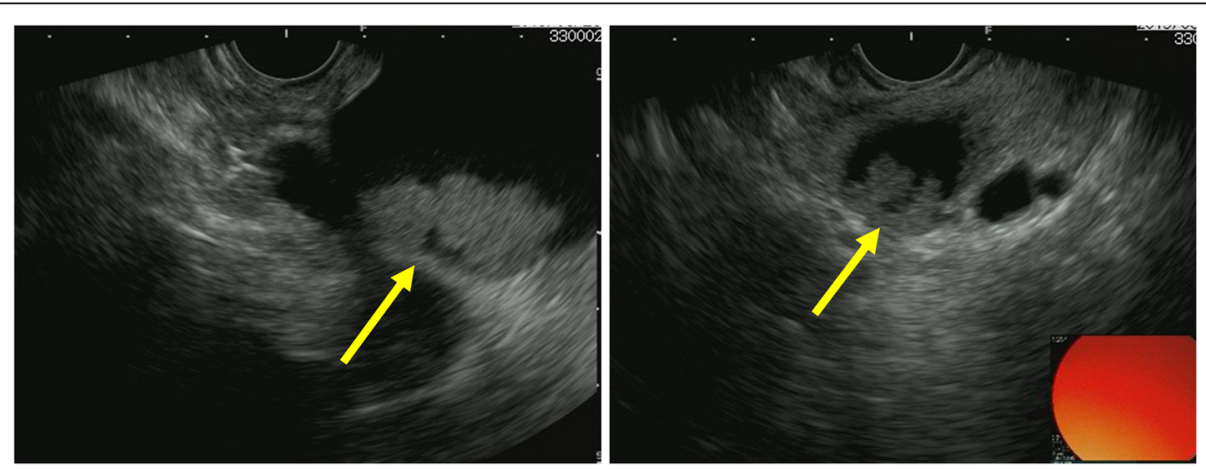

Fig. 2 Endoscopic ultrasonography (EUS) imaging findings. EUS revealed that the cystic tumor of the pancreatic head was a 35-mm solitary cyst with a 24-mm mural nodule, and the cystic tumor of the pancreatic tail was a 20-mm solitary cyst with a mural nodule 


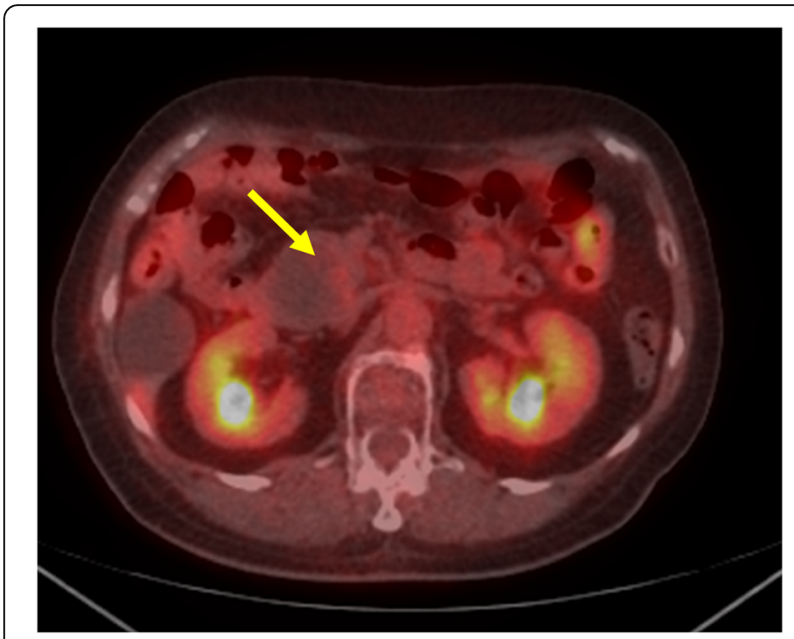

Fig. $3^{18}$ F-Fluorodeoxyglucose positron-emission tomography findings (FDG-PET). FDG-PET showed FDG uptake (SUV max 1.9) in the lesion at the pancreatic head. No evidence of metastasis from other organs was observed

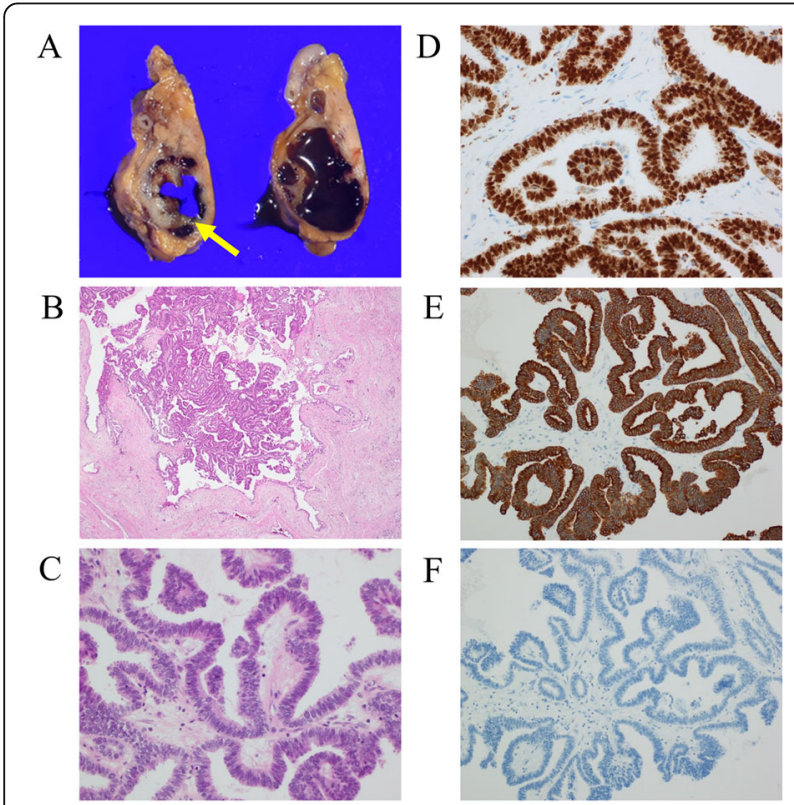

Fig. 4 Histopathological findings of pancreatic tumors. a Macroscopic findings showed that the tumor comprised of a cystic wall and mural nodule, which contained cystic fluid. b Hematoxylineosin staining $(\times 40)$. Papillary tumors were growing in large cysts. Tumor cells had no mucus in the cytoplasm and had different characteristics from intraductal papillary mucinous neoplasm. The papillary structure was similar to that of the previously resected lung cancer tissue. c Hematoxylin-eosin staining $(\times 200)$ showed the power field of a. d Multiplex immunohistochemical staining $(\times 400)$, using specific antibody cocktails of TTF-1 and Napsin A. Nuclear expression of TTF-1 and granular cytoplasmic expression of Napsin A were seen. e CK7 was positive by immunohistochemical staining. $\mathbf{f}$ CK20 did not present significant staining
According to these findings, we diagnosed the patient with metastatic pancreatic carcinoma from lung cancer. The postoperative course was good, and the patient was discharged 21 days after the operation. The patient did not received adjuvant therapy and had no recurrence for 6 months after pancreatectomy.

\section{Discussion}

Metastatic pancreatic tumors are reported to consist of renal cell carcinoma, breast cancer or colorectal cancer, and lung cancer, which accounts for $3 \%$ of all cases [1]. On the other hand, lung cancer metastasizes to the pancreas at a frequency of $13.8 \%$, and small cell carcinoma is the most frequent histological type [4].

In imaging diagnosis, metastatic pancreatic tumors are reported to reflect the features of primary lesions, and pancreatic metastases of renal cell carcinoma are relatively easy to diagnose as they are hypervascular tumors, like the primary tumor [5]. MPTLC is reported to have several features, such as excluding stenosis and semilunar disruption of the main pancreatic duct by endoscopic retrograde cholangiopancreatography or hypovascular tumors and ring-enhancing images by enhanced CT/MRI [2]. Rumancik et al. have argued that it is difficult to distinguish MPTLC from primary pancreatic cancer in diagnostic imaging [6]. Recently, several studies reported the feasibility of endoscopic ultrasound-guided fine-needle aspiration (EUS-FNA) to confirm diagnoses [7, 8]. Cystic MPTLC is extremely rare, with few reports in the literature. Ramirez et al. reported cystic MPTLC cases that showed metastasis 2 years after lobectomy for undifferentiated large-cell lung carcinoma [9].

IPMN is defined as a pancreatic tumor producing mucus with a papillary epithelial structure [10]. IPMN is classified as having worrisome features or high-risk stigmata from several malignant risk factors [11]. The 2017 revision of the International Association of Pancreatology consensus guidelines suggested that high-risk stigmata were obstructive jaundice, an enhancing mural nodule $\geq 5 \mathrm{~mm}$, and dilation of the main pancreatic duct to a diameter of $\geq 10 \mathrm{~mm}$. Because our case had features of enlargement, obstructive jaundice, and papillary nodules inside cystic lesions, it was difficult to distinguish MPTLC from IPMN with high-risk stigmata. Moreover, EUS-FNA was difficult to perform in this case due to cystic changes. In this patient, the case findings suggested a chronologically increasing cyst with the appearance of an enhancing mural nodule, and these were the basis for considering it IPMN with high-risk stigmata. On the other hand, it was untypical of IPMN that dilation of the main pancreatic duct was not observed with the increase in cyst size.

Reddy and Wolfgang proposed the following surgical indications for metastatic pancreatic tumors: (i) a 

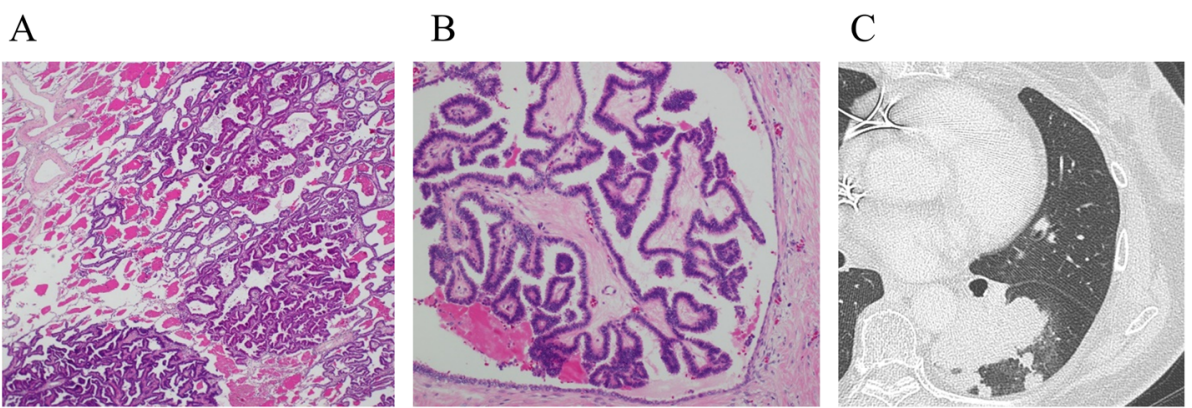

Fig. 5 Histopathological and computed tomography (CT) findings of lung adenocarcinoma that was resected 2 years before pancreatectomy.a Hematoxylin-eosin staining $(\times 40)$. This figure shows a part, including the boundary with the non-tumor part. Tumors formed papillary structures in some places, and there were also alveolar epithelial replacement growths. b Hematoxylin-eosin staining $(\times 200)$ showed the power field of $\mathbf{a}$. $\mathbf{c}$ Lung cancer had been detected at the left lower lobe as a solid tumor by CT

relatively better prognosis of the primary lesion, (ii) a controlled primary lesion, (iii) absence of multiple metastases, (iv) a resectable metastatic lesion, and (v) an operable condition of the patient [1]. A standard pancreatectomy with lymph node dissection is recommended to prevent recurrence. Although the resection of MPTLC was not recommended because of poor improvements in prognosis [12], Dietzek et al. reported good surgical indications for MPTLC with long intervals between initial therapy and recurrence [13]. Masetti et al. evaluated the prognoses of 234 pancreatic metastasis cases, and poor prognostic factors included being symptomatic, having multiple metastases, and incomplete resection in univariate analysis, and incomplete resection and melanoma in multivariate analysis [14]. Pancreatic metastasis of renal cell carcinoma had a significantly better prognosis than other cancers. MPTLCs have been treated with surgical resection in cases of metastases from adenocarcinoma or squamous cell carcinoma, but metastases from small cell carcinoma were mostly treated with chemotherapy.

In this case, adenocarcinoma was diagnosed as the primary lesion, which showed eosinophilic secretions pathologically. In the pancreatic lesions, no mucus was found in the cyst components or tumor cells, suggesting the accumulation of eosinophilic secretions in the tumor as in the primary lesion. These eosinophilic secretions and papillary nodules in the cyst exhibited a morphology that resembled that of IPMN with high-risk stigmata. Regarding the diagnosis, the histological and immunohistochemical similarities (TTF-1, Napsin A, and CK7 were positive) with lung cancer were comprehensively evaluated, and this tumor could be diagnosed as an MPTLC. Several pathways have been reported for metastatic pancreatic tumors, including direct invasion from surrounding organs, lymphatic metastasis to the peripancreatic lymph node, or hematogenous metastasis [15]. Because this case had no metastasis in 53 pieces of dissected lymph node, hematogenous metastasis is most likely the implicated pathway. As a characteristic clinical course of this case, the progression rate was rapid for IPMN. This case was labeled as metachronous metastasis, and radical resection was performed due to multiple lesions in the pancreas. Therefore, it is necessary to monitor for recurrence in the future carefully.

\section{Conclusion}

In this case, the cystic morphology was formed by eosinophilic secretions from tumor cells, and it was difficult to distinguish from IPMN with high-risk stigmata. We consider this case, based on the variable clinical findings, an extremely rare variant of MPTLC.

\section{Abbreviations}

MPTLC: Metastatic pancreatic tumor from lung cancer; IPMN: Intraductal papillary mucinous neoplasm; EUS-FNA: Endoscopic ultrasound-guided fine needle aspiration; FDG-PET: ${ }^{18}$ F-Fluorodeoxyglucose positron-emission tomography; CT: Computed tomography; MRI: Magnetic resonance imaging

\section{Acknowledgements}

None.

\section{Authors' contributions}

AW reported the case and wrote the manuscript. NH, KA, NK, TI, MT, NI, TY, and $\mathrm{KH}$ were involved in treating the patient and helped draft the manuscript. AS made diagnoses from the pathological findings. KS participated in critically revising the manuscript. All authors have read and approved the final manuscript.

\section{Funding}

No grant support or funding from public institutions or private enterprises was received for this case report.

\section{Availability of data and materials}

The dataset supporting the conclusions of this article is included in the article.

Consent for publication

Written informed consent was obtained from the patient for the publication of this case report and the accompanying images.

Competing interests

The authors declare that they have no competing interests. 


\section{Author details}

'Department of General Surgical Science, Division of Hepatobiliary and Pancreatic Surgery, Graduate School of Medicine, Gunma University, 3-39-15 Showamachi, Maebashi, Gunma 371-8511, Japan. ${ }^{2}$ Department of General Surgical Science, Division of Hepatobiliary and Pancreatic Surgery, Graduate School of Medicine, Gunma University, 3-39-22 Showamachi, Maebashi, Gunma 371-8511, Japan. ${ }^{3}$ Department of Innovative Cancer Immunotherapy, Gunma University, 3-39-15 Showamachi, Maebashi, Gunma 371-8511, Japan. ${ }^{4}$ Department of Pathology, Gunma University Hospital, 3-39-15 Showamachi, Maebashi, Gunma 371-8511, Japan. ${ }^{5}$ Division of Allergy and Respiratory Medicine, Integrative Center of Internal Medicine, Gunma University Hospital, 3-39-15 Showamachi, Maebashi, Gunma 371-8511, Japan.

Received: 20 March 2020 Accepted: 12 May 2020

Published online: 28 May 2020

\section{References}

1. Reddy S, Wolfgang CL. The role of surgery in the management of isolated metastases to the pancreas. Lancet Oncol. 2009;10:287-93.

2. Swensen T, Osnes M, Serck-Hanssen A. Endoscopic retrograde cholangiopancreatography in primary and secondary tumours of the pancreas. $\mathrm{Br}$ J Radiol. 1980;53:760-4.

3. James D. Brierley (Editor) MKGE, Christian Wittekind (Editor). TNM classification of malignant tumours, 8th edition. Wiley-Blackwell. 2016:272 Pages.

4. Maeno $T$, Satoh $H$, Ishikawa $H$, Yamashita $Y T$, Naito T, Fujiwara M, et al. Patterns of pancreatic metastasis from lung cancer. Anticancer Res. 1998;18: 2881-4.

5. McNichols DW, Segura JW, DeWeerd JH. Renal cell carcinoma: long-term survival and late recurrence. J Urol. 1981;126:17-23.

6. Rumancik WM, Megibow AJ, Bosniak MA, Hilton S. Metastatic disease to the pancreas: evaluation by computed tomography. J Comput Assist Tomogr. 1984:8:829-34

7. Fabbri C, Luigiano C, Maimone A, Tarantino I, Baccarini P, Fornelli A, et al. Endoscopic ultrasound-guided fine-needle biopsy of small solid pancreatic lesions using a 22-gauge needle with side fenestration. Surg Endosc. 2015; 29:1586-90.

8. Krishna SG, Bhattacharya A, Ross WA, Ladha H, Porter K, Bhutani MS, et al. Pretest prediction and diagnosis of metastatic lesions to the pancreas by endoscopic ultrasound-guided fine needle aspiration. J Gastroenterol Hepatol. 2015;30:1552-60.

9. Ramirez Plaza CP, Suarez Munoz MA, Santoyo Santoyo J, Perez Lara FJ, laria $M$, Raso L, et al. Pancreatic cystic metastasis from pulmonary carcinoma. Report of a case. Ann Ital Chir. 2001;72:95-9.

10. Suzuki Y, Atomi Y, Sugiyama M, Isaji S, Inui K, Kimura W, et al. Cystic neoplasm of the pancreas: a Japanese multiinstitutional study of intraductal papillary mucinous tumor and mucinous cystic tumor. Pancreas. 2004;28: 241-6.

11. Tanaka M, Fernandez-del Castillo C, Adsay V, Chari S, Falconi M, Jang JY, et al. International consensus quidelines 2012 for the management of IPMN and MCN of the pancreas. Pancreatology. 2012;12:183-97.

12. Crippa S, Angelini C, Mussi C, Bonardi C, Romano F, Sartori P, et al. Surgical treatment of metastatic tumors to the pancreas: a single center experience and review of the literature. World J Surg. 2006;30:1536-42.

13. Dietzek AM, Gupta SK, Kram HB, Wengerter KR, Veith FJ. Limb loss with patent infra-inguinal bypasses. Eur J Vasc Surg. 1990;4:413-7.

14. Masetti M, Zanini N, Martuzzi F, Fabbri C, Mastrangelo L, Landolfo G, et al. Analysis of prognostic factors in metastatic tumors of the pancreas: a singlecenter experience and review of the literature. Pancreas. 2010;39:135-43.

15. Ishigure K, Kanazumi N, Ohshima K, Kotake K, Sawada K, Nonami T, Nakao A. Report of three resected cases of pancreatic metastatic tumors. Jpn J Gastroenterol Surg. 2000;33:1686-90.

\section{Publisher's Note}

Springer Nature remains neutral with regard to jurisdictional claims in published maps and institutional affiliations.

\section{Submit your manuscript to a SpringerOpen ${ }^{\circ}$ journal and benefit from:}

- Convenient online submission

- Rigorous peer review

- Open access: articles freely available online

- High visibility within the field

- Retaining the copyright to your article

Submit your next manuscript at $\boldsymbol{\nabla}$ springeropen.com 